\title{
Difference between Right and Left Facial Surface Electromyography in Healthy People
}

\author{
Bo-Hyun Kim, ${ }^{1}$ Kyeong Han Kim ${ }^{D},{ }^{2}$ Lak-Hyung Kim, \\ Jong-Uk Kim, ${ }^{1}$ and Tae-Han Yook $\mathbb{1}^{1}$ \\ ${ }^{1}$ Department of Acupuncture \& Moxibustion Medicine, College of Korean Medicine, Woosuk University, Republic of Korea \\ ${ }^{2}$ Department of Preventive Medicine, College of Korean Medicine, Woosuk University, Republic of Korea \\ ${ }^{3}$ Department of Neuropsychiatry, College of Korean Medicine, Woosuk University, Republic of Korea \\ Correspondence should be addressed to Tae-Han Yook; nasiss@naver.com
}

Received 8 June 2018; Accepted 2 August 2018; Published 16 August 2018

Academic Editor: Sang-Hoon Shin

Copyright (C) 2018 Bo-Hyun Kim et al. This is an open access article distributed under the Creative Commons Attribution License, which permits unrestricted use, distribution, and reproduction in any medium, provided the original work is properly cited.

\begin{abstract}
Introduction. The study was to see whether there were differences in values of facial surface electromyography in subjects of good heath by muscles, age, and sex. Methods. It draws ratio between lower value and higher value (R-LV/HV) and asymmetry index (AI), based on root mean square (RMS) from measurement of facial surface electromyography (sEMG) in 154 people of healthy people (male:female $=70: 84$ ) aging between more than 20 and less than 70 . Results. For R-LV/HV, it averages $81.70 \pm 14.60 \%$ on frontalis muscle, $73.74 \pm 19.12 \%$ on zygomaticus muscle, and $79.72 \pm 14.77 \%$ on orbicularis oris muscle. With analysis of the AI average was $10.87 \pm 10.14 \%$ on frontalis muscle, $16.71 \pm 14.79 \%$ on zygomaticus muscle, and $12.10 \pm 10.05 \%$ on orbicularis oris muscle. Both values were statistically significant in three parts of muscles as shown. Both of R-LV/HV and AI show no statistically significant difference on age and sex ( $p>0.05)$. Conclusions. It could provide basic data for the future diagnosis of facial nerve palsy patients by measuring facial sEMG values for healthy people.
\end{abstract}

\section{Introduction}

Facial paralysis has a major symptom of atonia paralysis on the facial muscle and was a disease with accompanying symptoms, such as decreased taste, hearing impairment, saliva secretion, and tear reduction [1]. Various hypotheses for causes of facial paralysis include viral infection, ischemic vascular disease-causing paralysis, vascular disorders due to diabetes, multiple neuritis, autoimmune disease, and cold exposure, but no hypothesis yet provides a clear explanation as to what causes facial paralysis [2]. It has been reported that $20-30$ persons every 100,000 in population annually experience facial paralysis [3]. As of 2015, there were 8,511 cases of facial paralysis reported in Korea [4].

Diagnostic techniques for patients with facial paralysis include House-Brackmann scale, Yanagihara grading system, and Sunnybrook facial grading system, which were performed on naked eyes $[5,6]$, and digital infrared thermal imaging, or DITI, electroneurography (ENoG), nerve excitability test (NET) or electromyography (EMG), and surface electromyography (sEMG) were utilized as diagnostic instruments [7].

Among other things sEMG was a kind of EMG diagnostic instrument that quantitatively measures electric signals for muscle movements. In general, EMG was measuring the electromyogram of a single muscle by inserting a needle, while sEMG was a noninvasive mechanism using surficial electrodes and was advantageous in conducting the overall assessment of facial movements, not just movements of a single muscle. Because of its strength, sEMG has been widely used as an assessing instrument of facial paralysis and was an index that has significance in determining facial states and degree of recovery with ratio between lower value and higher value $(\mathrm{R}-\mathrm{LV} / \mathrm{HV})$, or asymmetry index (AI) [8].

Although there have been many studies on sEMG of patents with facial paralysis, less studies have been reported on whether the indexes were different by sex, age, or parts of the body. In some study [9], there was a difference in facial 
sEMG values according to sex, but there was a limit of 40 small subjects. This study was to measure facial surface sEMG in subjects of good health aging between 20 s and 60 s to see any difference in the values depending on sex, age, or parts of the body.

\section{Methods}

2.1. Participants. The study mobilizes people aging older than 20 and less than 70 between September 13, 2016, and March 1, 2017. Prospective participants in the study were asked if they have the existing disease or have been administered with related medications through the basic examination and preliminary medical examination. Participants who were not subject to exclusion were selected as the final subjects. A total of 154 people wish to participate in the study and no subjects fall within exclusion. The final subjects include 154 people, 70 males and 84 females. Exclusion criteria are as follows.

(1) Persons with anamnesis of stroke

(2) Persons currently suffering from diseases associated with facial paralysis or with anamnesis of such diseases

(3) Persons with facial disease or body disease that could affect other facial electromyography

(4) Persons who have been given medications or experienced activity within one week that will affect measurement of sEMG

(5) Persons having discomfort with facial muscle movements due to plastic surgery or facial operation

(6) Persons who may have a displacement because they continuously use facial muscles in occupation (such as performer of brass instrument)

(7) Persons with facial asymmetry of Grade 2 on HouseBrackmann scale through naked eye assessment

(8) Other cases of exclusion a researcher would determine inadequate

\subsection{Study Implementation}

2.2.1. Medical Device. For sEMG implementation, a fourchannel adopting electromyography system QEMG-4 XL (manufactured by Laxtha Co. Ltd., Korea) was used, while QEMG-4 XL (version1.0 Neuromedi Inc.) was used for measurement. For electrode sensor, AM530 active electromyography system (manufactured by Laxtha Co. Ltd., Korea) was used.

2.2.2. $R-L V / H V$. A higher value from the left and right measurements in the total of 154 subjects was set on numerator and a lower value regarding as denominator. The formula is as follows.

$$
\text { Ratio }(\%)=\frac{\text { EMG }(\text { low value side })}{\text { EMG }(\text { High Value side })} \times 100
$$

2.2.3. AI. AI is difference in the values divided by the sum of the values. In the study, the difference value obtained after deducting $\mathrm{R}-\mathrm{LV} / \mathrm{HV}$, among root mean square (RMS) on the left and right measurements, was then divided by the sum of RMS values on the left and right parts to obtain AI. Higher AI means significant difference in RMS values on the left and right muscles. The formula is as follows.

$$
\begin{aligned}
& \text { Asymmetry Index (\%) } \\
& =\frac{\text { EMG (high value side) }- \text { EMG (low value side) }}{\text { EMG (high value side) }- \text { EMG (low value side) }} \\
& \times 100
\end{aligned}
$$

2.3. Measurement. The placement of electrode was made in parallel to muscular fibers of frontalis muscle, zygomaticus muscle, and orbicularis oris muscle. A first electrode was placed on the left, while a second electrode was place on the right. To eliminate factors reducing any skin resistance to the sEMG measuring signals, the measurement site was cleaned with medical alcohol cotton and its surfaces were to be completely dried before the electrode was placed. The real measurement was conducted when a subject fully learned how to move after preliminary measurement. The measurement of sEMG uses signal processing of root mean square (RMS). A relaxation time for one-time electromyography signal measurement was set on five seconds and tension time on three seconds. Gain index was between the ranges of -1463 and 1463 . Each test was repeated with three measurements and the average measurement of the three measurements was used as a measurement value. Subjects were required to take a 10 -minute rest and then return to the measurement of sEMG by having a disposable electrode placed on acupuncture points of frontalis muscle, zygomaticus muscle, and orbicularis oris muscle after being introduced how to move muscles at each acupuncture point.

2.3.1. Frontalis Muscle (Yangbaek (GB14)). The acupuncture point of Yangbaek (GB14) is located directly above pupil by a finger joint from the eyebrow [8]. For movements of frontalis muscle and Yangbaek (GB14), a subject is required to move the eyebrows to form wrinkles on his forehead (Figure 1).

2.3.2. Zygomaticus Muscle (Gwonyo (SI18)). The acupuncture point of Gwonyo (SI18) is located sunken at ends of Yegol below Myeonpigol [8]. For movements of zygomaticus muscle and Gwonyo (SI18), a subject is required to pull angular upward and outside (Figure 1).

2.3.3. Orbicularis Oris Muscle (Seungjang (CV24)). The acupuncture point of Seungjang (CV24) is located sunken at edges of the lips [8]. Orbicularis oris muscle is located by a finger joint from both sides of Seungjang (CV24). For movements of orbicularis oris muscle, a subject is required to pucker lips forward to hold out (Figure 1). 


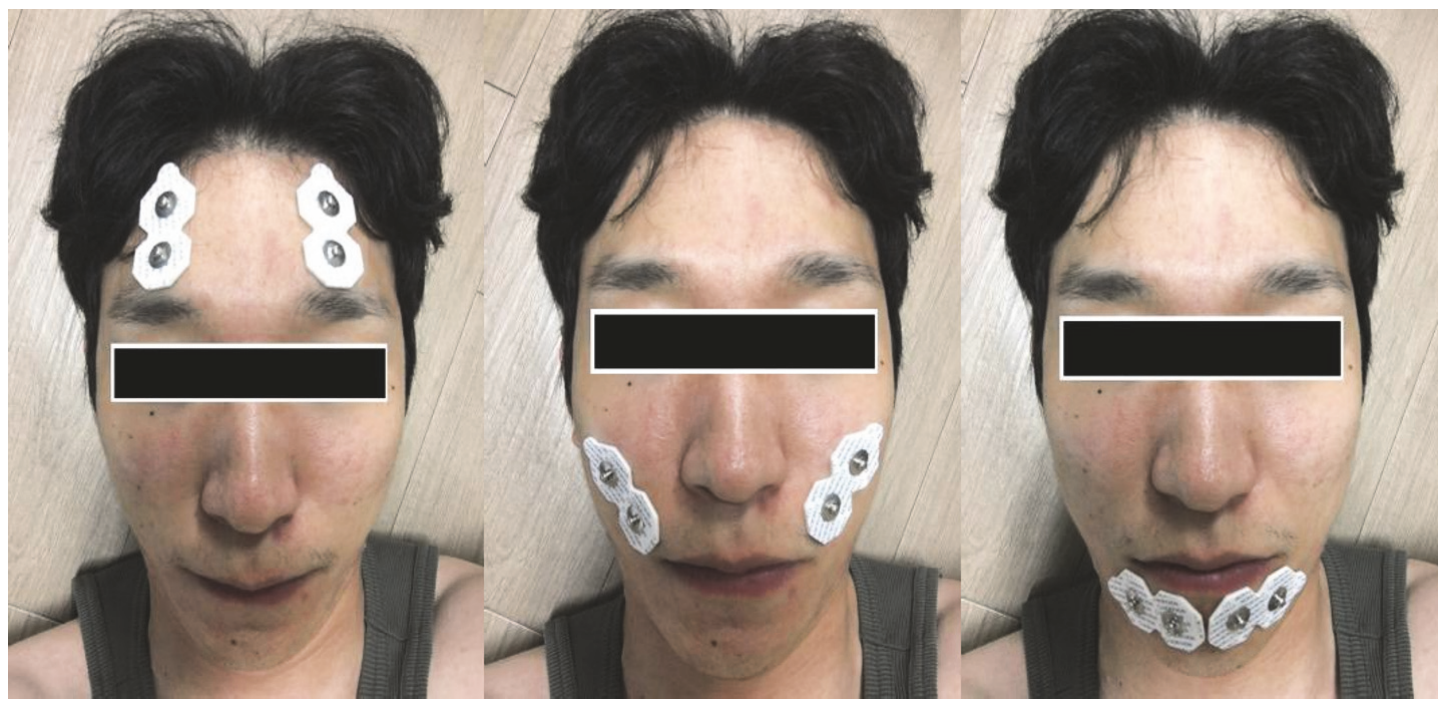

FIGURE 1: Attachment site of frontalis muscle, zygomaticus muscle, and orbicularis oris muscle.

2.4. Statistical Analysis. For statistics of research results, SPSS Statistics 22.0 version 64 bit edition (IBM, USA) was used and all the measurements were indicated in mean \pm SD. For sex comparison, paired t-test was conducted and one-way analysis of variance (ANOVA) followed by a post hoc Scheffe test was used to compare muscle and age. When $p$-value was less than 0.05 , this was interpreted to have a statistically significance, and all the values were rounded up from the third decimal place.

2.5. Ethics. The study was approved by Institutional Review Board, IRB of Jeonju Oriental Hospital in affiliation with Woosuk University (No. WSOH IRB 1610-06).

\section{Results}

3.1. Sociodemographic Characteristics. The gathered group of males and female were in their 20s and 60s. For age distribution of the people gathered, 31 people were in the range of between older than 20 and younger than 30, 29 people were in the range of between older than 30 and younger than 40,37 people were in the range of between older than 40 and younger than 50, 29 people were in the range of between older than 50 and younger than 60 and 28 people were in the range of between older than 60 and younger than 70 . The average height of male was $171.56 \pm 5.29 \mathrm{~cm}$ and average weight was $72.34 \pm 8.74 \mathrm{~kg}$. The average height of female was $160.25 \pm 5.22 \mathrm{~cm}$ and average weight was $58.17 \pm 8.80$ (Table 1 ).

3.2. Difference in $R-L V / H V$ and $A I$ between Muscles. The total of 154 subjects on the three parts have R-LV/HV and AI were measured (Table 2). For R-LV/HV, it averages on $81.70 \pm 14.60 \%$ on frontalis muscle, $73.74 \pm 19.12 \%$ on zygomaticus muscle, and $79.72 \pm 14.77 \%$ on orbicularis oris muscle. When they were compared using one-way ANOVA, R-LV/HV on each part shows statistically significant difference $(\mathrm{p}<0.001)$. Frontalis muscle value was higher than zygomaticus muscle value and orbicularis oris muscle value was higher than zygomaticus muscle value. However, it was no significant difference between frontalis muscle value and orbicularis oris muscle value.

For AI, it averages on $10.87 \pm 10.14 \%$ on frontalis muscle, $16.71 \pm 14.79 \%$ on zygomaticus muscle, and $12.10 \pm 10.05 \%$ on orbicularis oris muscle (Table 2). When they were compared using one-way ANOVA, the asymmetry index on each part shows statistically significant difference $(p<0.001)$. Frontalis muscle value was lower than zygomaticus muscle value and orbicularis oris muscle value was lower than zygomaticus muscle value. However, there was no significant difference between frontalis muscle value and orbicularis oris muscle value.

3.3. Difference in $\mathrm{R}-\mathrm{LV} / \mathrm{HV}$ and $\mathrm{AI}$ between Ages. For R$\mathrm{LV} / \mathrm{HV}$, it has its highest of $84.05 \pm 15.54 \%$ in 50 s on frontalis muscle and its lowest of $78.75 \pm 19.01 \%$ in 30 s. It has its highest of $79.76 \pm 17.87 \%$ in 30 s on zygomaticus muscle and its lowest of $69.13 \pm 18.03 \%$ in 60 s. It has its highest of $81.26 \pm 12.63 \%$ in 30 s on orbicular oris muscle and its lowest of $76.52 \pm 16.33 \%$ in 20s. There was no significant difference between age in RLV/HV ( $>>0.05)$ (Table 3).

For AI, it has its highest of $13.22 \pm 13.11 \%$ in 30 s on frontalis muscle and its lowest of $9.17 \pm 7.64 \%$ in 20 s. It has its highest of $19.88 \pm 16.14 \%$ in 60 s on zygomaticus muscle and its lowest of $12.45 \pm 12.57 \%$ in 30 s. It has its highest of $14.29 \pm 11.20 \%$ in 20 s on orbicular oris muscle and its lowest of $10.88 \pm 8.19 \%$ in 30s. There was no significant difference between age in AI (p>0.05) (Table 3).

3.4. Difference in $\mathrm{R}-L V / H V$ and $A I$ between Male and Female. For $\mathrm{R}-\mathrm{LV} / \mathrm{HV}$, it averages on $82.37 \pm 15.29 \%$ in 70 males on frontalis muscle, $75.40 \pm 19.32 \%$ on zygomaticus muscle, and $80.00 \pm 15.50 \%$ on orbicularis oris muscle. It averages on $81.15 \pm 14.07 \%$ in 84 females on frontalis muscle, $72.35 \pm 18.96 \%$ on zygomaticus muscle, and $79.49 \pm 14.21 \%$ on orbicularis oris 
TABLE 1: Demographic characteristics of 154 subjects.

\begin{tabular}{cccc}
\hline Classification & Male & Female & Total \\
\hline Age (person) & & & 84 \\
Total & 70 & 17 & 31 \\
$20 \mathrm{~s}$ & 14 & 14 & 29 \\
$30 \mathrm{~s}$ & 15 & 23 & 37 \\
$40 \mathrm{~s}$ & 14 & 15 & 29 \\
$50 \mathrm{~s}$ & 14 & 15 & 28 \\
$60 \mathrm{~s}$ & 13 & $160.25 \pm 5.22$ & $165.39 \pm 7.70$ \\
height $(\mathrm{cm})$ & $171.56 \pm 5.29$ & $58.17 \pm 8.80$ & $64.61 \pm 11.25$ \\
weight $(\mathrm{kg})$ & $72.34 \pm 8.74$ & & \\
\hline
\end{tabular}

TABLE 2: Difference R-LV/HV and AI between muscles.

\begin{tabular}{|c|c|c|c|c|c|c|}
\hline classification & $\begin{array}{c}\text { FM } \\
\text { Mean } \pm \text { SD (\%) }\end{array}$ & $\begin{array}{c}\mathrm{ZM} \\
\text { Mean } \pm \text { SD (\%) }\end{array}$ & $\begin{array}{c}\text { OM } \\
\text { Mean } \pm \text { SD (\%) }\end{array}$ & $\mathrm{F}$ & $\mathrm{p}$ & Post Hoc \\
\hline R-LV/HV & $81.70 \pm 14.60$ & $73.74 \pm 19.12$ & $79.72 \pm 14.77$ & 9.965 & $<.001$ & $\begin{array}{l}\mathrm{FM}>\mathrm{ZM} \\
\mathrm{ZM}<\mathrm{OM} \\
\mathrm{FM}=\mathrm{OM}\end{array}$ \\
\hline AI & $10.87 \pm 10.14$ & $16.71 \pm 14.79$ & $12.10 \pm 10.05$ & 10.365 & $<.001$ & $\begin{array}{l}\mathrm{FM}<\mathrm{ZM} \\
\mathrm{ZM}>\mathrm{OM} \\
\mathrm{FM}=\mathrm{OM}\end{array}$ \\
\hline
\end{tabular}

* FM: frontalis muscle; ZM: zygomaticus muscle; OM: orbicularis oris muscle.

muscle. When they were compared using paired t-test, the RLV/HV by sex shows no statistically significant difference on all the three parts $(\mathrm{p}>0.05)$ (Table 4$)$.

For AI, it averages on $10.56 \pm 10.89 \%$ in 70 males on frontalis muscle, $15.62 \pm 14.86 \%$ on zygomaticus muscle, and $12.03 \pm 10.84 \%$ on orbicularis oris muscle. It averages on $11.13 \pm 9.53 \%$ in 84 females on frontalis muscle, $17.62 \pm 14.77 \%$ on zygomaticus muscle, and $12.16 \pm 9.40 \%$ on orbicularis oris muscle. When they were compared using paired t-test, the R$\mathrm{LV} / \mathrm{HV}$ by sex shows no statistically significant difference on all the three parts $(\mathrm{p}>0.05)$ (Table 4$)$.

\section{Discussion}

Most widely used methods for assessing facial paralysis include House-Brackmann scale, Yanagihara grading system, and Sunnybrook facial grading system which were methods of assessment with facial movements in patients as well as utilization of diagnosis devices such as DITI, NET, ENoG, EMG, and sEMG [10].

Among these methods, sEMG was a test that measures action potential by attaching electrodes on surfaces of the skin. Muscle forms a figure of the body by being attached to skins and the skeletal system and supervises exercise of the whole body that moves the skeleton system. When muscle retraction occurs, this simultaneously triggers motor impulse signal on motor cortex in the brain which was continuously transmitted into nerves of each motor unit through motor neurons of the spinal cord $[11,12]$. When these nerve impulses were brought to neuromuscular junction, this would cause an electric transmission along muscular fibers to sarcolemma in both directions, which was called motor unit action potential, or MUAP [13]. Electrodiagnosis was defined as capturing, amplifying, and recording the electric action in muscles and was based on measurements of occurrence, mobilization, and propagation of these action potentials to be displayed on screen. The electrodes of the sEMG were divided into pole electrodes and surface electrodes based on measuring sites and convenience, and the surface electrode was used to alleviate pains of subjects when the measurement was conducted [14]. Unlike other methods, the sEMG was regarded as a relatively simple procedure not requiring artificial electric stimulus or noninvasive stimulus and thus has a potential for popularity, especially for the facial parts because a patient shows no resistance to its use on them. A study on surficial sEMG was emerging as the new paradigm in the field of rehabilitation for muscular and skeletal disease. There have been brisk study efforts in overseas going on the sEMG, including types of electrode and location of placement $[15,16]$.

For literature review on related studies on sEMG conducted in Asian countries such as Korea, Japan, and China, there were less than 10 studies on the sEMG in each country as of 2012 and there was the only clinical literature on patients with facial paralysis accompanying coordination movements [17]. Although there have been attempts to interpret results of the sEMG test on patients with facial paralysis by associating with the naked eye test, it was insufficient to represent the sEMG test with the smaller number of samples of 21 people. Another ongoing study on the sEMG measurements of 20 males and 40 females with good health when they move their facial parts was designed to draw biological electric features of the local specimen with good health but shows its limitation in that it fails to provide no classification other than 
TABLE 3: Average of R-LV/HV and AI between ages.

\begin{tabular}{|c|c|c|c|c|c|c|c|}
\hline classification & $\begin{array}{c}20 \mathrm{~s} \\
\text { Mean } \pm \text { SD }(\%)\end{array}$ & $\begin{array}{c}30 \mathrm{~s} \\
\text { Mean } \pm \text { SD (\%) }\end{array}$ & $\begin{array}{c}40 s \\
\text { Mean } \pm \text { SD (\%) }\end{array}$ & $\begin{array}{c}50 s \\
\text { Mean } \pm \text { SD (\%) }\end{array}$ & $\begin{array}{c}60 s \\
\text { Mean } \pm \text { SD (\%) }\end{array}$ & F & $\mathrm{p}$ \\
\hline \multicolumn{8}{|l|}{$\mathrm{R}-\mathrm{LV} / \mathrm{HV}$} \\
\hline FM & $83.99 \pm 11.99$ & $78.75 \pm 19.01$ & $81.65 \pm 13.32$ & $84.05 \pm 15.54$ & $79.86 \pm 12.72$ & .781 & .539 \\
\hline $\mathrm{ZM}$ & $76.11 \pm 14.99$ & $79.77 \pm 17.87$ & $72.66 \pm 18.93$ & $71.00 \pm 24.27$ & $69.14 \pm 18.03$ & 1.440 & .224 \\
\hline $\mathrm{OM}$ & $76.53 \pm 16.33$ & $81.26 \pm 12.64$ & $80.65 \pm 15.43$ & $79.71 \pm 16.29$ & $80.44 \pm 12.86$ & .487 & .745 \\
\hline \multicolumn{8}{|l|}{ AI } \\
\hline FM & $9.18 \pm 7.65$ & $13.23 \pm 13.12$ & $10.76 \pm 9.31$ & $9.61 \pm 11.71$ & $11.76 \pm 8.35$ & .770 & .546 \\
\hline $\mathrm{ZM}$ & $14.41 \pm 10.34$ & $12.46 \pm 12.57$ & $17.32 \pm 14.05$ & $19.58 \pm 19.34$ & $19.89 \pm 16.15$ & 1.413 & .232 \\
\hline $\mathrm{OM}$ & $14.30 \pm 11.20$ & $10.89 \pm 8.19$ & $11.60 \pm 10.75$ & $12.28 \pm 11.38$ & $11.40 \pm 8.17$ & .528 & .715 \\
\hline
\end{tabular}

* FM: frontalis muscle; ZM: zygomaticus muscle; OM: orbicularis oris muscle.

TABLE 4: Average of each muscles in R-LV/HV and AI between male and female.

\begin{tabular}{lcccc}
\hline classification & $\begin{array}{c}\text { Male }(\mathrm{N}=70) \\
\text { Mean } \pm \text { SD (\%) }\end{array}$ & $\begin{array}{c}\text { Female (N=84) } \\
\text { Mean } \pm \text { SD (\%) }\end{array}$ & $\begin{array}{c}\text { Total (N=154) } \\
\text { Mean } \pm \text { SD (\%) }\end{array}$ & $\mathrm{t}$ \\
\hline R-LV/HV & & & & \\
FM & $82.37 \pm 15.29$ & $81.15 \pm 14.07$ & $81.70 \pm 14.60$ & .512 \\
ZM & $75.40 \pm 19.32$ & $72.35 \pm 18.96$ & $73.74 \pm 19.12$ & .983 \\
OM & $80.00 \pm 15.50$ & $79.49 \pm 14.21$ & $79.72 \pm 14.77$ & .213 \\
AI & & & & .327 \\
FM & $10.56 \pm 10.89$ & $11.13 \pm 9.53$ & $10.87 \pm 10.14$ & .831 \\
ZM & $15.62 \pm 14.86$ & $17.62 \pm 14.77$ & $16.71 \pm 14.79$ & .728 \\
OM & $12.03 \pm 10.84$ & $12.16 \pm 9.40$ & $12.10 \pm 10.05$ & -.834 \\
\hline
\end{tabular}

* FM: frontalis muscle; ZM: zygomaticus muscle; OM: orbicularis oris muscle.

classification by males and females as well as with the smaller number of specimen $[18,19]$.

For sEMG analysis, the widely used statistical analysis was used. RMS was a method that analyzes amplitude of signal shown in sEMG. The analysis was available to measure the number of motor units activated and firing rate as it represents an increasing aspect of the signal amplitude when the muscle retracts and a decreasing aspect when muscle fatigue occurs [20]. It also has a significance in its utilization when $\mathrm{R}-\mathrm{LV} / \mathrm{HV}$, Al determine conditions of the facial parts and degree of recovery. No report has been made as to whether the indexes of subjects with good health have a difference in sex, age, or parts of the body.

The authors in the study obtained $\mathrm{R}-\mathrm{LV} / \mathrm{HV}, \mathrm{Al}$ from measurements of the facial surface sEMG in the subjects aged between 20 s and 60s and with good health to clarify if the values have any difference in sex, age, or parts of the body. The study was based on a total of 154 males and females who aged between 20 s and 60s and with good health gathered starting from September 13, 2016, to May 1, 2017. The selected subjects were required to take a 10 -minute rest and introduced to learn how to move muscles of each acupuncture point to measure sEMG by placing a disposable electrode on acupuncture points of frontalis muscle, zygomaticus muscle, and orbicularis oris muscle. To measure orbicularis oris muscle, it selects a lower orbicularis oris muscle by a finger joint from both sides of Seungjanghyeol and this is based on results found in the study of Kim et al. [21] that orbicularis oris muscle has a higher measurement on the lower orbicularis oris muscle than on the higher orbicularis oris muscle.

The total of 154 subjects on the three parts have R$\mathrm{LV} / \mathrm{HV}$ and AI were measured. For R-LV/HV, it averages on $81.70 \pm 14.60 \%$ on frontalis muscle, $73.74 \pm 19.12 \%$ on zygomaticus muscle, and $79.72 \pm 14.77 \%$ on orbicularis oris muscle. When they were compared using one-way ANOVA, R$\mathrm{LV} / \mathrm{HV}$ on each part shows statistically significant difference. Frontalis muscle value was higher than zygomaticus muscle value and orbicularis oris muscle value was higher than zygomaticus muscle value. However, it was no significant difference between frontalis muscle value and orbicularis oris muscle value. For AI, it averages on $10.87 \pm 10.14 \%$ on frontalis muscle, $16.71 \pm 14.79 \%$ on zygomaticus muscle, and $12.10 \pm 10.05 \%$ on orbicularis oris muscle. When they were compared using one-way ANOVA, the asymmetry index on each part shows statistically significant difference. Frontalis muscle value was lower than zygomaticus muscle value and orbicularis oris muscle value was lower than zygomaticus muscle value. However, it was no significant difference between frontalis muscle value and orbicularis oris muscle value.

For R-LV/HV, it has its highest of $84.05 \pm 15.54 \%$ in 50 s on frontalis muscle and its lowest of $78.75 \pm 19.01 \%$ in 30 s. It has its highest of $79.76 \pm 17.87 \%$ in 30 s on zygomaticus muscle and its lowest of $69.13 \pm 18.03 \%$ in 60 s. It has its highest of $81.26 \pm 12.63 \%$ in 30 s on orbicular oris muscle and its lowest of $76.52 \pm 16.33 \%$ in 20 s. There was no significant 
difference between age in R-LV/HV. For AI, it has its highest of $13.22 \pm 13.11 \%$ in 30 s on frontalis muscle and its lowest of $9.17 \pm 7.64 \%$ in 20 s. It has its highest of $19.88 \pm 16.14 \%$ in 60 s on zygomaticus muscle and its lowest of $12.45 \pm 12.57 \%$ in 30 s. It has its highest of $14.29 \pm 11.20 \%$ in 20 s on orbicular oris muscle and its lowest of $10.88 \pm 8.19 \%$ in 30 s. There was no significant difference between age in AI.

For $\mathrm{R}-\mathrm{LV} / \mathrm{HV}$, it averages on $82.37 \pm 15.29 \%$ in 70 males on frontalis muscle, $75.40 \pm 19.32 \%$ on zygomaticus muscle, and $80.00 \pm 15.50 \%$ on orbicularis oris muscle. It averages on $81.15 \pm 14.07 \%$ in 84 females on frontalis muscle, $72.35 \pm 18.96 \%$ on zygomaticus muscle, and $79.49 \pm 14.21 \%$ on orbicularis oris muscle. When they were compared using paired t-test, the R-LV/HV by sex shows no statistically significant difference on all the three parts. For AI, it averages on $10.56 \pm 10.89 \%$ in 70 males on frontalis muscle, $15.62 \pm 14.86 \%$ on zygomaticus muscle, and $12.03 \pm 10.84 \%$ on orbicularis oris muscle. It averages on $11.13 \pm 9.53 \%$ in 84 females on frontalis muscle, $17.62 \pm 14.77 \%$ on zygomaticus muscle, and $12.16 \pm 9.40 \%$ on orbicularis oris muscle. When they were compared using paired t-test, the R-LV/HV by sex shows no statistically significant difference on all the three parts.

As found in the results stated above, individual differences in RMS values of the sEMG in subjects of good health were large and the same large differences were found in range of standard deviation, while $\mathrm{R}-\mathrm{LV} / \mathrm{HV}$ or $\mathrm{AI}$ in subjects of good health shows relatively certain range of values. This comes down to the conclusion as stated in Lee et al. [19] that RMS values were not suitable for determining conditions of subjects, rather comparisons using R-LV/HVs and AI values can be more reasonable method to determine whether a subject has a normal condition or not. Based on the conclusion, the results in this study can be utilized to assess abnormality of facial muscles and determine whether they fall within the normality.

\section{Conclusion}

The study analyzes R-LV/HV and AI with measurements of surface electromyography or sEMG on acupunctures points such as frontalis muscle, zygomaticus muscle, and orbicularis oris in a total of 154 subjects with good health.

(1) For R-LV/HV, it averages $81.70 \pm 14.60 \%$ on frontalis muscle, $73.74 \pm 19.12 \%$ on zygomaticus muscle, and $79.72 \pm 14.77 \%$ on orbicularis oris muscle. With analysis of the AI average was $10.87 \pm 10.14 \%$ on frontalis muscle, $16.71 \pm 14.79 \%$ on zygomaticus muscle, and $12.10 \pm 10.05 \%$ on orbicularis oris muscle.

(2) R-LV/HV was significance in three parts of muscles $(\mathrm{FM}>\mathrm{ZM}, \mathrm{ZM}<\mathrm{OM}, \mathrm{FM}=\mathrm{OM})$. And $\mathrm{AI}$ also was significance difference in muscles $(\mathrm{FM}<\mathrm{ZM}, \mathrm{ZM}>\mathrm{OM}$, $\mathrm{FM}=\mathrm{OM}$ )

(3) Both of R-LV/HV and AI showed no statistically significant difference on age and sex.

In subjects of good health, no difference was found in terms of $\mathrm{R}-\mathrm{LV} / \mathrm{HV}$ and $\mathrm{AI}$ either by sex or by age. It was anticipated that the results in this study will be utilized to determine diagnosis, prognosis, and recovery in the future.

\section{Data Availability}

The data used to support the findings of this study may be released upon application to the IRB of Jeonju Oriental Hospital in affiliation with Woosuk University.

\section{Conflicts of Interest}

The authors have declared no conflicts of interest.

\section{Authors' Contributions}

Bo-Hyun Kim and Kyeong Han Kim equally contributed to this study.

\section{Acknowledgments}

This research achievement was conducted with assistance from the National Research Fund (NRF) subsidized by the Ministry of Education in 2015 (NRF-2015R1D1A3A01019492) and the Traditional Korean Medicine R\&D Program funded by the Ministry of Health \& Welfare through the Korea Health Industry Development Institute (KHIDI) (HB16C0028).

\section{References}

[1] Korean Acupuncture \&amp; Moxibustion Medicine Society Textbook Publish Committee Compilation. The Acupuncture and Moxibustion medicine. Paju:Jipmoondang2012:625-9.

[2] H. J. Hong, Analysis of Affecting Factors for Prognosis of Patients with Bell's Palsy, Department of Medicine TheGraduate School, Yonsei University, 2006.

[3] Korean society of otorhinolaryngology. Otorhinolaryngology. Otorhinolaryngology. Seoul: chokak. 2005:209-11.

[4] National Health Insurance Service. Facial nerve palsy patient [Internet]. Seoul: Author;2015[Cited 2016 September 01].

[5] M. B. Kim, J. H. Kim, S. H. Shin, H. J. Yoon, and W. S. Ko, "A study of facial nerve grading system," The Journal of Korean Oriental Medical Ophthalmology, Otolaryngology and Dermatology, vol. 20, no. 3, pp. 147-160, 2007.

[6] J. W. Lee, S. A. Kwon, M. J. Kim et al., "A Study of Facial Palsy Sequelae and Evaluating Scale," The Journal of Korean Acupuncture Moxibustion Society, vol. 28, no. 2, pp. 75-87, 2011.

[7] G. H. Koo, "Facial Nerve Palsy," Korean journal of pain, vol. 9, no. 1, pp. 14-15, 1996.

[8] The Acupuncture and Moxibustion Medicine Textbook Compilation Committee. Acupuncture Medicine. Seoul :Hanmibook. 2016. 299-661.

[9] HG. Lee, DJ. Jung, and YM. Choi, "A Study of Surface Elctromyography Measurement of Facial Muscles in normal Person," The Acupuncture, vol. 31, no. 2, pp. 51-63, 2014.

[10] D. Dumitru, N. E. Walsh, and L. D. Porter, "Leslie D Porter. Electrophysiologic evaluation of the facial nerve in Bell's palsy," American Journal of Physical Medicine \& Rehabilitation, vol. 67, no. 4, pp. 137-144, 1988. 
[11] H. W. Jeong, Undonghakchongseol-Total Opinion of Kinesiology, Published by Mokgwa, 2002, Published by Mokgwa To.

[12] T. U. Kim, "Muscular exercise and muscle fatigue," Journal of Korea Spots Research, vol. 18, no. 6t, pp. 229-240, 2007.

[13] J. H. Cho, J. S. Lee, and S. S. Kim, "A Study of the meridian muscle electrography for the clinical application," Journal of Oriental Rehabilitation Medicine, vol. 15, no. 4, pp. 89-104, 2005.

[14] H. B. Kim, Y. H. Park, and S. S. Bae, "Clinical application of electromyography and nerve conduction study," Journal of the Korean Society of Physical Medicine, vol. 10, no. 1, pp. 199-212, 1998.

[15] B. G. Lapatki, D. F. Stegeman, and I. E. Jonas, "A surface EMG electrode for the simultaneous observation of multiple facial muscles," Journal of Neuroscience Methods, vol. 123, no. 2, pp. 117-128, 2003.

[16] L. Mesin, R. Merletti, and A. Rainoldi, "Surface EMG: The issue of electrode location," Journal of Electromyography \& Kinesiology, vol. 19, no. 5, pp. 719-726, 2009.

[17] H. G. Lee, J. G. Im, D. J. Jung, J. U. Kim, L. H. Kim, and T. H. Yook, "Comparative review on oriental medicine study utilized surface electromyography in Korea," Journal of Korean Acupuncture Moxibustion Medicine Society, vol. 30, no. 1, pp. 2334, 2013.

[18] JU. Kim, HG. Lee, DJ. Jung et al., "A Study on the Correlation between Surface Electromyography and Assessment Scale for Facial Palsy," Journal of Korean Acupuncture \& Moxibustion Medicine Society, vol. 30, no. 5, pp. 107-116, 2013.

[19] H. G. Lee, D. J. Jung, Y. M. Choi et al., "A Study of Surface Electromyography Measurement of Facial Muscles in Normal Person," Journal of Korean Acupuncture \&amp; Moxibustion Medicine Society, vol. 31, no. 2, pp. 51-63, 2014.

[20] K. J. Han and B. K. Choi, "Comparison of the Surface Electromyographic Signal of Progressive Resistance Increase and Progressive Resistance Decrease Exercise," Journal of the Korean Society of Physical Medicine, vol. 20, no. 1, pp. 11-16, 2008.

[21] J. Y. Kim, B. H. Kim, H. B. Kim, T. H. Yook, and J. U. Kim, "A Study of Surface Electromyography Measurement of Orbicularis oris motion in Healthy People," Journal of Korean Acupuncture \&amp; Moxibustion Medicine Society, vol. 33, no. 4, pp. 93-100, 2016. 


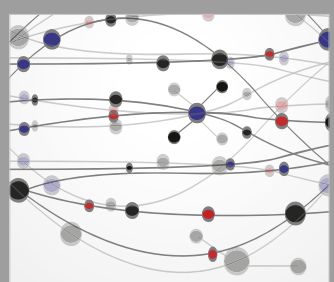

The Scientific World Journal
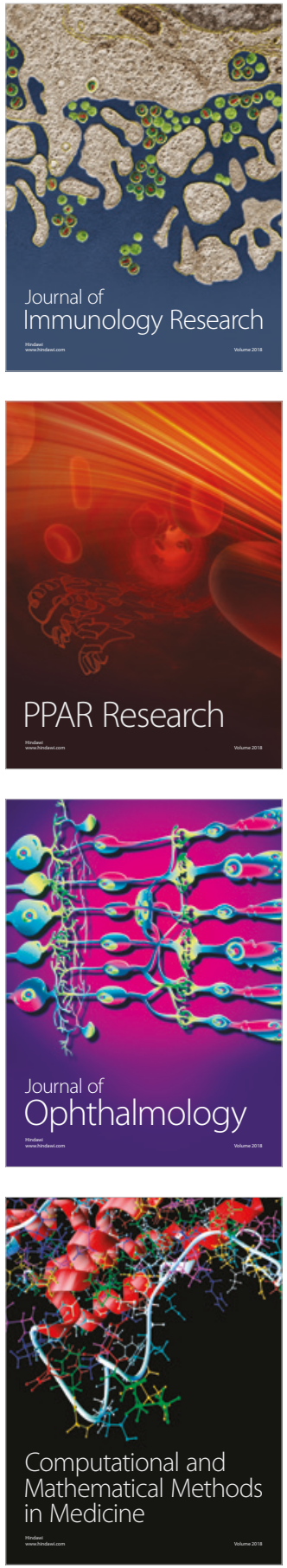

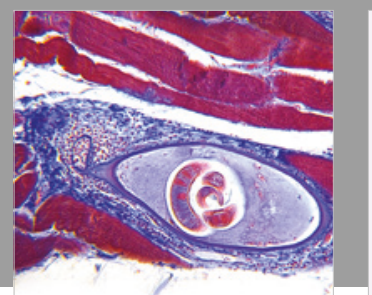

Gastroenterology Research and Practice

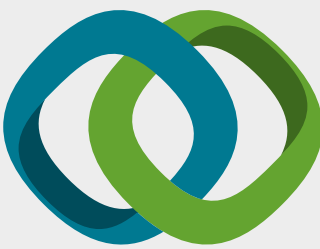

\section{Hindawi}

Submit your manuscripts at

www.hindawi.com
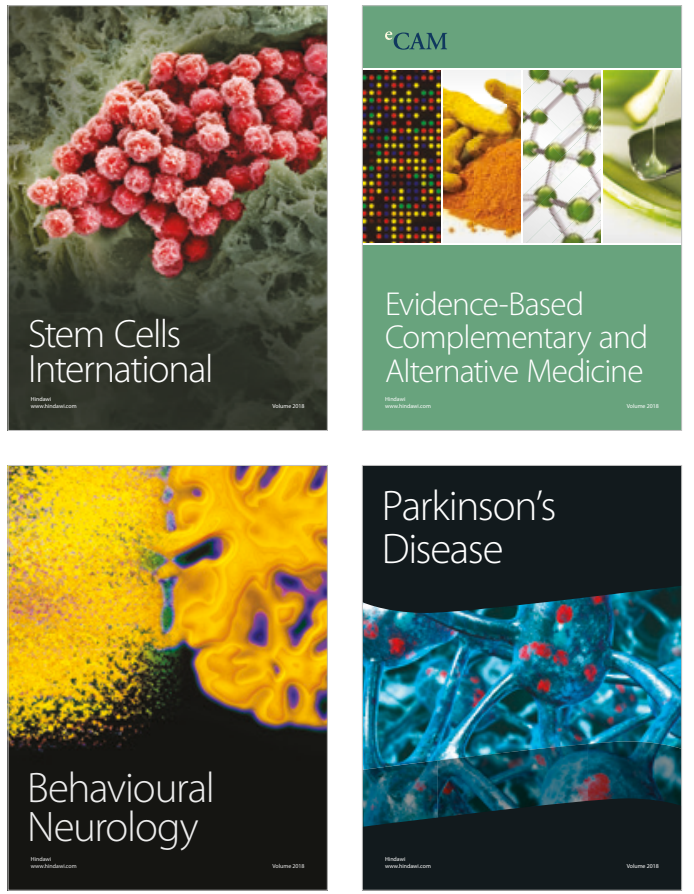

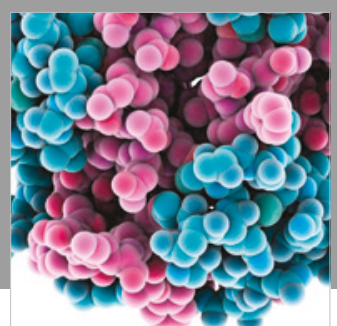

ournal of

Diabetes Research

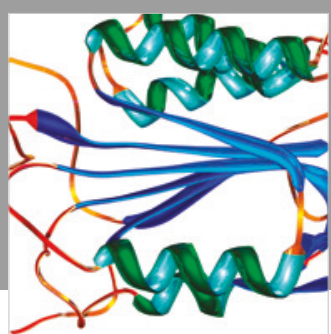

Disease Markers
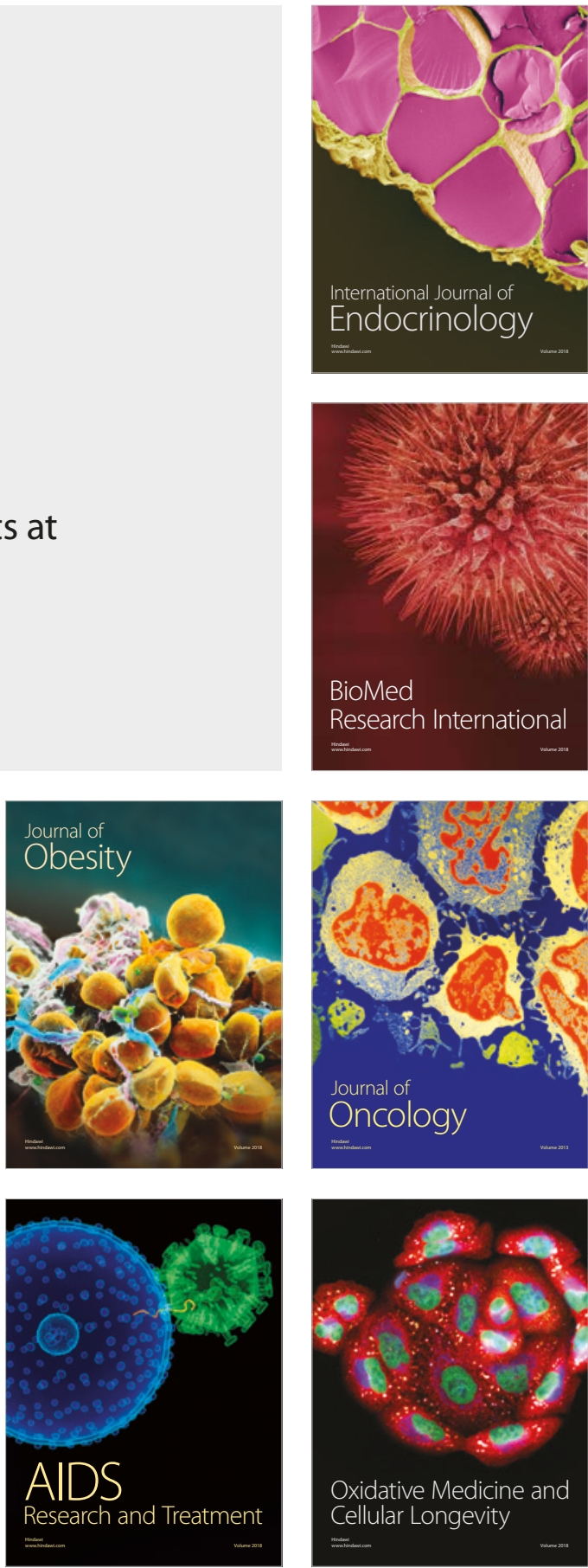